\title{
Robust UAV Mission Planning
}

\author{
Lanah Evers *,1,2,3, Twan Dollevoet ${ }^{3}$, Ana Isabel Barros ${ }^{1,2}$, and Herman Monsuur ${ }^{2}$ \\ ${ }^{1} \mathrm{TNO}$ - Defense, Security and Safety \\ ${ }^{2}$ Netherlands Defence Academy \\ ${ }^{3}$ Econometric Institute, Erasmus University Rotterdam
}

Econometric Institute Report EI 2011-07

February 25, 2011

\begin{abstract}
Unmanned Areal Vehicles (UAVs) can provide significant contributions to information gathering in military missions. UAVs can be used to capture both full motion video and still imagery of specific target locations within the area of interest. In order to improve the effectiveness of a reconnaissance mission, it is important to visit the largest number of interesting target locations possible, taking into consideration operational constraints related to fuel usage between target locations, weather conditions and endurance of the UAV. We model this planning problem as the well-known orienteering problem, which is a generalization of the traveling salesman problem. Given the uncertainty in the military operational environment, robust planning solutions are required. As such, our model takes into account uncertainty in the fuel usage between targets (for instance due to weather conditions) as well as uncertainty in the importance of visiting specific target locations. We report results using different uncertainty sets that specify the degree of uncertainty against which any feasible solution will be protected. We also compare the probability that a solution is feasible for the robust solution on one hand and the solution found with average fuel usage and expected value of information on the other. In doing so, we show how the sustainability of a UAV mission can be significantly improved.
\end{abstract}

\section{Introduction}

Current military operations pose new challenges for Intelligence, Surveillance and Reconnaissance missions (ISR) as mentioned by Maj. Gen. Michael Flynn in [19]. Effective and flexible approaches to data collection are among the critical elements of military operations. Following the successful employment in Desert Storm (see [23]), Unmanned Aerial Vehicles (UAVs) have become an important asset in tactical reconnaissance. Depending on the type of information to be gathered, the use of UAVs can be preferred over collection assets (for instance, in harsh environments, or when information collection is too tedious for human operators, or when quicker response times are required). As such, they are often used in reconnaissance missions to capture both motion and still imagery of potential targets and areas of interest on which up-to-date information is required. Such locations might include important infrastructure, possible locations of Improvised Explosive Devices (IEDs), and insurgent locations. The goal of the UAV mission is thus to gather as much information as possible given operational constraints related to the UAV endurance capabilities (available fuel capacity since refueling is not permitted during flight). Obviously, some locations

\footnotetext{
*Corresponding author: Lanah.Evers@tno.nl
} 
can be more relevant than others in terms of information gathering for the military operation, and therefore priorities are usually assigned to the locations. In order to optimize data collection the UAV should fly a tour including target locations of higher priority, starting and ending at the UAV recovery point. Hence, this problem can be formulated as the Orienteering Problem (OP), wherein the set of target locations correspond to nodes, the profits on the nodes model the target location priorities, the arcs model the flight path from one target location to the other, and the fuel requirement for such a flight path is given by the cost on the associated arc. The depot represents the recovery point of the UAV. This generalization of the Traveling Salesman Problem (TSP) does not require that all the nodes have to be visited and considers a profit associated with each node. The profit is collected only if a node is visited. The goal is to find a tour of maximum profit, starting and ending at the depot that is feasible with regard to a cost constraint that restricts the total costs of the arcs that are included in the tour.

Royset and Reber [18] use this model to plan UAV missions, aiming to locate insurgents placing IEDs and already placed IEDs. To obtain the profit values, they use the data from an IED prediction tool. The tool provides the expected number of IEDs for each grid location. Not only are the profits modeled as deterministic parameters in their model, the same is done for the cost, which represent the expected fuel consumption. On the other hand, Porto [16] considers the allocation of multiple reconnaissance UAVs to acquire temporally variable, time-differential intelligence data (i.e. targets have to be revisited on a periodic basis to obtain time differential images). In this specific case the tours of the multiple UAVs are optimized over and around the targets of interest cooperatively, i.e., desired imaging schedules are met via a combination of assets. In the proposed model, Porto also considers explicitly the probability of survival of the UAVs as a function of the time the UAV spends traveling through threat zones.

Since UAVs operate in a dynamic and uncertain environment, effective UAV mission plans should be able to deal with environment changes and with changing expectations. In fact, weather circumstances like wind have a great impact on the fuel consumption of the UAV (real fuel consumption will differ from the expected fuel consumption). Also, the expected priority assigned to target locations often varies (priorities change as the mission evolves or due to unexpected events). Furthermore, since replanning costs can be significant, especially in large systems it is important to generate UAV tour plans that are robust. In this paper, we explicitly address uncertainty in the input parameters of the described UAV mission planning. Hence, we assume that both the costs on the arcs as well as the profits on the nodes are uncertain. As such, we introduce the Robust Orienteering Problem (ROP) and we will come up with solutions that are robust against both weather conditions and variations of the target priorities. Our robustness approach is two folded. Uncertainty related to the fuel constraint (a strict operational requirement) is modeled using absolute robustness that ensures that the UAV tour can be completed for all possible realizations of fuel consumption considered within the so-called uncertainty set. On the other hand, we use a globalized robustness approach to handle profit uncertainty. In the globalized robust approach two uncertainty sets are defined. It provides full protection against uncertainty in the first set, while uncertainty in the second set is only covered to a certain extend. To the best of our knowledge, no earlier research on the problem described by the ROP exists. In fact, Bertucelli et al. [6] incorporate target uncertainty in the target value by assuming that the realization of each target value lies within a certain predefined interval. They consider the situation in which UAVs need to be assigned to a set of targets in the area. Their approach differs in two aspects from our research. First of all, Bertucelli et al. consider a task assignment problem of multiple UAVs in which each UAV is assigned to one target. We, on the other hand, consider a routing problem of one UAV visiting multiple targets. Secondly, Bertucelli et al. do not take fuel constraints into account.

This paper is structured as follows. In the following section we will provide some background on robust optimization. In Section 3 we will formally describe the nominal OP. Next we will introduce the ROP in which we explicitly model data uncertainty. In Section 5 we illustrate the effects of incorporating both types of robustness by means of a case study. We conclude in Section 6 with some remarks on how the results of this case study relate to a real-life UAV planning problem. 


\section{Robust optimization}

In this section we will provide some background on robust optimization theory that will be used further in the modeling of our UAV planning problem. In a standard linear programming problem $\max _{x}\left\{c^{T} x: A x \leq b\right\}$ (LP) the parameters described by $A, b$ and $c$ are deterministic. In reality however, some or all of these parameters might be uncertain. Therefore, the solution to the deterministic LP might turn out to be infeasible in reality. Robust optimization aims to overcome this issue by taking into account data uncertainty already at the modeling stage.

In 1973 Soyster [20] was the first to incorporate uncertainty in an LP. One of the characteristics of the Soyster approach is that it provides very conservative solutions, since resulting solutions can deal with all worst-case realizations at the same time. This rather conservative approach sacrifices optimality for the nominal problem, while the probability of all parameters reaching their worst-case value at the same time is low in practice. However, in the late nineties, Kouvelis and $\mathrm{Yu}$ [12], Ben-Tal and Nemirovski [3], [4] and El Ghaoui et al. [8], [9] developed new robust optimization frameworks for respectively Integer Programming (Kouvelis and Yu [12]) and Convex Programming that deal better with this issue.

In the remainder of this section we will focus on the robust optimization of LPs in the framework as provided by Ben-Tal et al. [2] and we will mention how some approaches applied by other authors are related to this framework. Ben-Tal et al. consider the situation where the realization of an uncertain parameter lies in a symmetric interval. For example, when the maximum deviation of element $(i, j)$ in $A$ is defined by $\sigma_{i j}$, realization $a_{i j}$ can be expressed by

$$
a_{i j}=\overline{a_{i j}}+\sigma_{i j} \zeta_{i j}
$$

where $\overline{a_{i j}}$ is the nominal value and $\zeta_{i j} \in[-1,1]$. The so-called robust counterpart of the LP provides a solution that is feasible for all realizations of $\zeta$ within a predefined uncertainty set $U$. The set $U$ can for example be defined as an intersection of balls:

$$
U=\left\{\zeta \in \mathbf{R}^{n}:\|\zeta\|_{s} \leq \rho_{s} \forall s \in S\right\}=\bigcap_{s \in S} B_{s}^{|A|}\left(\rho_{s}\right),
$$

where we defined

$$
B_{s}^{n}\left(\rho_{s}\right)=\left\{\zeta \in \mathbf{R}^{n}:\|\zeta\|_{s} \leq \rho_{s}\right\}
$$

to simplify the notation. The norm $\|\cdot\|_{s}$ determines the shape of the ball, while $\rho_{s}$ defines its size. $S$ is an index set. We will discuss some examples of uncertainty sets $U$.

When the goal is to find a solution that is protected against all parameters reaching their worst-case value at the same time, we need to define $S$ as a singleton with $s=\infty$ and $\rho_{\infty}=1$, because this gives

$$
U=B_{\infty}^{n}(1)=\left\{\zeta \in \mathbf{R}^{n}:\|\zeta\|_{\infty} \leq 1\right\}=\left\{\zeta \in \mathbf{R}^{n}: \max _{i=1, \ldots, n} \zeta_{i} \leq 1\right\}
$$

This type of uncertainty is called 'box-uncertainty'. Note that a solution that is feasible for all realizations in this uncertainty set, is equivalent to a solution that satisfies the Soyster formulation.

As discussed in the Introduction, Bertucelli et al. address the problem of assigning AUVs to targets. The approach they use in order to find an assignment that is robust with respect to uncertainty in the target value, is related to the use of the uncertainty set just defined. More specifically, Bertucelli et al. maximize the total value of the targets, decreased by $\mu$ times its maximum deviation from the nominal target value, where $0 \leq \mu \leq 1$. As such, the approach on robustness applied by Bertucelli et al. is a special case within the framework of Ben-Tal et al, where the sum of worst-case realizations over the target uncertainty set $U=B_{\infty}^{n}(\mu)$ is maximized.

Finally, consider the example where we aim to find a solution that remains feasible if up to $\rho_{1}$ uncertain parameters change from their nominal value. This could be implemented in the problem formulation by defining

$$
U=B_{1}^{n}\left(\rho_{1}\right)=\left\{\zeta \in \mathbf{R}^{n}:\|\zeta\|_{1} \leq \rho_{1}\right\}=\left\{\zeta \in \mathbf{R}^{n}: \sum_{i=1}^{n}\left|\zeta_{i}\right| \leq \rho_{1}\right\}
$$


Note that if we would only apply this constraint on $\zeta$, we also allow for realizations of the individual parameters $\zeta_{i}$, outside of their predefined interval. Therefore, we could choose the uncertainty set $U$ against which any feasible solution is protected, as the intersection $B_{1}^{n}\left(\rho_{1}\right) \cap B_{\infty}^{n}(1)$. This type of uncertainty is called 'budget uncertainty', which is the type of uncertainty set used by Bertsimas and $\operatorname{Sim}[5]$.

\section{The nominal orienteering problem}

In this section we will give a formal description of the OP in which all input parameters are assumed to be deterministic. The $\mathrm{OP}$ was introduced by Tsiligrides [21] and was shown to be strongly NP-Hard by Laporte and Martello [13]. An exact algorithm for the OP was proposed by Fischetti et al. [11]. The OP belongs to the class of TSPs with profits, of which an overview is given by Feillet et al. [10]. A recent survey on the OP is given by Vansteenwegen et al. [22]. The OP can be formulated in several different ways (see for example three formulations of the team orienteering problem given by Poggi et al. [15]). In this section, we will present a formulation that is based on the Miller-Tucker-Zemlin formulation [14] for the TSP. In this formulation, both the number of variables and the number of constraints are polynomial in the number of targets.

\subsection{Formulation of the nominal OP}

Denote $N$ for the set of targets and $|N|$ for its cardinality. Denote the depot location by vertex $s \notin N$. For notational convenience, define $N^{+}=N \cup\{s\}$. With each target $i \in N$ we associate a target (profit) value $p_{i}$. We formulate the OP on a complete graph $G=\left(N^{+}, A\right)$ with $|N|+1$ vertices. With each arc $(i, j)=a \in A$ we associate a cost $f_{i j}$ representing the expected fuel consumption between vertex $i$ and $j$. The fuel capacity of the UAV is denoted by $F$. We introduce a binary decision variable $x_{i j}$ for every $\operatorname{arc}(i, j) \in A . x_{i j}$ is 1 if arc $(i, j)$ is used in the tour. An auxiliary variable $u_{i}$ is introduced to denote the position of vertex $i$ in the tour. The goal is to find a tour of maximum profit, feasible with respect to the fuel constraint, which starts and ends at the depot. Based on these definitions, the formulation of the OP is the following:

$$
\text { (OP) } \max \sum_{i \in N} p_{i} \sum_{j \in N^{+} \backslash\{i\}} x_{i j},
$$

such that

$$
\begin{array}{cr}
\sum_{i \in N} x_{s i}=\sum_{i \in N} x_{i s}=1, & \\
\sum_{i \in N^{+} \backslash\{k\}} x_{i k}=\sum_{i \in N^{+} \backslash\{k\}} x_{k i} \leq 1, & \\
\sum_{(i, j)=a \in A} f_{i j} x_{i j} \leq F, & \\
u_{i}-u_{j}+1 \leq\left(1-x_{i j}\right)|N|, & \forall i, j \in N, \\
1 \leq u_{i} \leq|N|, & \forall i \in N, \\
x_{i j} \in\{0,1\}, & \forall(i, j) \in A .
\end{array}
$$

Constraint (6) guarantees that the tour starts and ends at the depot. Constraints (7) are the flow conservation constraints and ensure that a vertex is visited at most once. Constraint (8) is the capacity constraint. Finally, Constraints (9) prevent the construction of subtours.

\section{The robust orienteering problem}

In this section we introduce a version of the OP in which uncertainty is taken into account, the Robust Orienteering Problem (ROP). More specifically, we will explicitly consider uncertainty in 
the costs of the arcs, as well as in the profits associated to the nodes. The corresponding robust UAV mission problem will therefore explicitly model both uncertainty in the fuel consumption (costs of the arcs) and in the priority of the targets (profits of the nodes). We will formulate and solve this new version of the OP by applying techniques from the framework provided by Ben-Tal et al. We will make use of very recent results by Ben-Tal and den Hertog [1] on incorporating Fenchel duality [17] in the general framework of robust optimization. The robust counterpart of simple uncertainty sets like box uncertainty is relatively easy to derive. The robust counterpart of polyhedral uncertainty can be derived using standard duality theory as described in Chapter 1 of [2]. Instead of using such derivations for each uncertainty set separately, the new theory by Ben-Tal and den Hertog allows us to define a general formulation of the ROP for any given uncertainty set.

Denote the expected fuel consumption from target $i$ to target $j$ by $\overline{f_{i j}}$ and the expected profit of target $i$ by $\overline{p_{i}}$. We assume that the realizations of these parameters lie in the intervals $\left[\overline{f_{i j}}-\sigma_{i j}^{f}, \overline{f_{i j}}+\sigma_{i j}^{f}\right]$ and $\left[\overline{p_{i}}-\sigma_{i}^{p}, \overline{p_{i}}+\sigma_{i}^{p}\right]$ respectively.

\subsection{Absolute robustness in fuel constraint}

For later convenience, we rewrite the realizations of the fuel consumption as

$$
f_{i j}=\overline{f_{i j}}+\sigma_{i j}^{f} \zeta_{i j}
$$

where $\zeta_{i j} \in[-1,1]$. As uncertainty set, we allow an arbitrary intersection of balls: we define $Z^{f}$, the set of possible realizations of $\zeta$, by

$$
Z^{f}=\left\{\zeta \in \mathbf{R}^{|A|}:\|\zeta\|_{s} \leq \rho_{s} \forall s \in S\right\}=\bigcap_{s \in S} B_{s}^{|A|}\left(\rho_{s}\right),
$$

with $B_{s}^{n}\left(\rho_{s}\right)$ as defined in Section 2. In Section 5.4, we will explicitly describe the uncertainty sets that we used in our computational experiments.

A solution for the ROP is absolute robust against the uncertainty in the fuel consumption if it satisfies Constraint 8 for all realizations $\zeta \in Z^{f}$. That is, if it holds that

$$
\sum_{(i, j) \in A}\left(\overline{f_{i j}}+\sigma_{i j}^{f} \zeta_{i j}\right) x_{i j} \leq F \quad \forall \zeta \in Z^{f}
$$

which is equivalent to

$$
\sum_{(i, j) \in A} \overline{f_{i j}} x_{i j}+\max _{\zeta \in Z^{f}} \sum_{(i, j) \in A} \sigma_{i j}^{f} \zeta_{i j} x_{i j} \leq F .
$$

In order to determine the second term of Equation 15, first define $f(\zeta, x)=\sum_{(i, j) \in A} \sigma_{i j}^{f} \zeta_{i j} x_{i j}$ and consider the indicator function

$$
\delta\left(\zeta \mid Z^{f}\right)= \begin{cases}0, & \text { if } \zeta \in Z^{f} \\ \infty, & \text { elsewhere }\end{cases}
$$

Then, the second term of Equation 15 can be rewritten as

$$
\max _{\zeta \in Z^{f}} \sum_{(i, j) \in A} \sigma_{i j}^{f} \zeta_{i j} x_{i j}=\max _{\zeta \in \mathbf{R}^{|A|}}\left\{f(\zeta, x)-\delta\left(\zeta \mid Z^{f}\right)\right\} .
$$

By Fenchel duality [17] Equation 16 is equivalent to

$$
\min _{d \in \mathbf{R}^{|A|}}\left\{\delta^{*}\left(d \mid Z^{f}\right)-f_{*}^{1}(d, x)\right\},
$$

where we use $g^{*}$ and $g_{*}$ respectively to denote the convex and the concave conjugate of $g: \mathbf{R}^{n} \rightarrow \mathbf{R}$ with dom $g=\{x \mid g(x)<\infty\}$, defined by

$$
g^{*}(d)=\sup _{x \in \operatorname{dom}}\left\{d^{T} x-g(x)\right\} \text { and }
$$




$$
g_{*}(d)=\inf _{x \in \operatorname{dom} g}\left\{d^{T} x-g(x)\right\} \text { respectively. }
$$

For $f_{*}^{1}$, which is the convex conjugate of $f$ with respect to the first variable $(\zeta)$, we find

$$
\begin{aligned}
f_{*}^{1}(d, x) & =\inf _{\zeta \in \mathbf{R}^{|A|}}\left\{d^{T} \zeta-\sum_{(i, j) \in A} \sigma_{i j}^{f} \zeta_{i j} x_{i j}\right\} \\
& =\inf _{\zeta \in \mathbf{R}^{|A|}} \sum_{(i, j) \in A}\left(d_{i j}-\sigma_{i j}^{f} x_{i j}\right) \zeta_{i j}= \begin{cases}0, & \text { if } d_{i j}=\sigma_{i j}^{f} x_{i j} \quad \forall(i, j) \in A ; \\
-\infty, & \text { elsewhere. }\end{cases}
\end{aligned}
$$

Therefore, Equation 17 boils down to

$$
\min _{d \in \mathbf{R}^{|A|}}\left\{\delta^{*}\left(d \mid Z^{f}\right)-f_{*}^{1}(d, x)\right\}=\delta^{*}\left(\sigma^{f} \otimes x \mid Z^{f}\right),
$$

where $\otimes$ denotes the operation resulting in a vector that contains the element-wise multiplications of its arguments: $\left(\sigma^{f} \otimes x\right)_{i j}=\sigma_{i j}^{f} x_{i j}$. It therefore suffices to evaluate the dual function $\delta^{*}$ at $\sigma^{f} \otimes x$. By definition,

$$
\delta^{*}\left(d \mid Z^{f}\right)=\max _{\zeta \in Z^{f}}\left\{d^{T} \zeta\right\}=\max \left\{d^{T} \zeta:\|\zeta\|_{s} \leq \rho_{s} \forall s \in S\right\} .
$$

This can be viewed as a conic optimization problem. By conic duality, it holds that

$$
\delta^{*}\left(d \mid Z^{f}\right)=\min \left\{\sum_{s \in S} \rho_{s} \tau_{s}: \sum_{s \in S} y_{s}=-d,\left\|y_{s}\right\|_{s}^{*} \leq \tau_{s},\left(y_{s}, \tau_{s}\right) \in \mathbf{R}^{|A|+1} \forall s \in S\right\},
$$

where $y \mapsto\|y\|^{*}$ denotes the dual norm, defined by $\|y\|^{*}=\sup \left\{y^{T} d:\|d\| \leq 1\right\}$. Replacing $y_{s}$ by $-y_{s}$ and eliminating $\tau_{s}$ for all $s \in S$, we obtain

$$
\delta^{*}\left(d \mid Z^{f}\right)=\min \left\{\sum_{s \in S} \rho_{s}\left\|y_{s}\right\|_{s}^{*}: \sum_{s \in S} y_{s}=d, y_{s} \in \mathbf{R}^{|A|} \forall s \in S\right\} .
$$

Substituting this into Equation 19, we find that $x$ is robust against uncertainty in the fuel consumption, if there exist vectors $y_{s} \in \mathbf{R}^{|A|}$, such that

$$
\begin{aligned}
\sum_{(i, j) \in A} \overline{f_{i j}} x_{i j}+\sum_{s \in S} \rho_{s}\left\|y_{s}\right\|_{s}^{*} & \leq F, \\
\sum_{s \in S} y_{s} & =\sigma^{f} \otimes x .
\end{aligned}
$$

In the special case that $S=\{s\}$ is a singleton, the above simplifies to

$$
\sum_{(i, j) \in A} \overline{f_{i j}} x_{i j}+\rho_{s}\left\|\sigma^{f} \otimes x\right\|_{s}^{*} \leq F .
$$

\subsection{Globalized robustness in profit objective}

To take the uncertainty of the profits into account, we first introduce an auxiliary decision variable $t$. Then we add the constraint

$$
t \leq \sum_{i \in N} p_{i} \sum_{j \in N^{+} \backslash\{i\}} x_{i j}
$$

to the formulation of the OP. The objective is now to maximize $t$. We rewrite the realizations of the profits as

$$
p_{i}=\overline{p_{i}}+\sigma_{i}^{p} \zeta_{i},
$$


where $\zeta_{i} \in[-1,1]$. Our goal is to optimize the worst case profit, where we consider the worst case over all realizations $\zeta \in Z^{p}$. However, we will also consider profits in the larger set $\Omega^{p} \supset Z^{p}$. For these profits, we allow for a slight loss in profit. This approach is called globalized robustness. We will now first introduce notation and define the constraints that a robust counterpart solution should satisfy. Consider the uncertainty sets

$$
\Omega^{p}=\left\{\zeta:\|\zeta\|_{A} \leq \rho_{A}\right\} \quad \text { and } \quad Z^{p}=\left\{\zeta:\|\zeta\|_{B} \leq \rho_{B}\right\},
$$

and the distance function

$$
\operatorname{dist}(\zeta, Z)=\min _{\zeta^{\prime} \in Z}\left\|\zeta-\zeta^{\prime}\right\|_{C}
$$

Now consider a constraint

$$
z_{0}(x)+z(x)^{T} \zeta \leq \alpha(\operatorname{dist}(\zeta, Z)) \quad \forall \zeta \in \Omega,
$$

where $\alpha(\cdot)$ is an increasing function with $\alpha(0)=0$. Ben-Tal and den Hertog [1] derived that $\left(z, z_{0}\right)$ is a globalized robust counterpart solution if and only if there exists a vector $v$ such that $z, z_{0}, v$ satisfy

$$
z_{0}+\rho_{A}\|v\|_{A}^{*}+\rho_{B}\|v-z\|_{B}^{*}+\alpha^{*}\left(\|v-z\|_{C}^{*}\right) \leq 0 .
$$

For our profit objective we require that for all $\zeta \in \Omega^{p}$ it holds that

$$
t-\alpha\left(\operatorname{dist}\left(\zeta, Z^{p}\right)\right) \leq \sum_{i \in N} p_{i} \sum_{j \in N^{+} \backslash\{i\}} x_{i j} .
$$

We can rewrite this constraint matching the structure of Constraint 29 as

$$
t-\sum_{i \in N} \overline{p_{i}} \sum_{j \in N^{+} \backslash\{i\}} x_{i j}-\sum_{i \in N} \sigma_{i}^{p} \zeta_{i} \sum_{j \in N^{+} \backslash\{i\}} x_{i j} \leq \alpha\left(\operatorname{dist}\left(\zeta, Z^{p}\right)\right) .
$$

For $a(s)=\beta s$, the dual function $\alpha^{*}$ can be computed easily. It is given by

$$
\alpha^{*}(d)= \begin{cases}0, & \text { if } d \leq \beta \\ \infty, & \text { otherwise }\end{cases}
$$

We see that the term $\alpha^{*}\left(\|v-z\|_{C}^{*}\right)$ reduces to the extra constraint $\|v-z\|_{C}^{*} \leq \beta$. Applying now the result given by Constraint 30 for $\alpha(s)=\beta s$, we find that solution to the OP is globalized robust against uncertainty in the profit values if and only if

$$
\begin{array}{r}
t-\sum_{i \in N} \overline{p_{i}} \sum_{j \in N^{+} \backslash\{i\}} x_{i j}+\rho_{A}\|v\|_{A}^{*}+\rho_{B}\left\|v+\left(\sigma^{p} \odot x\right)\right\|_{B}^{*} \leq 0, \\
\left\|v+\left(\sigma^{p} \odot x\right)\right\|_{C}^{*} \leq \beta,
\end{array}
$$

where $\odot$ denotes the operation resulting in a vector that contains the elements $\left(\sigma^{p} \odot x\right)_{i}=$ $\sum_{j \in N^{+} \backslash\{i\}} \sigma_{i}^{p} x_{i j}$. For later convenience, we introduce an auxiliary variable $w \in \mathbf{R}^{|N|}$ that satisfies

$$
w=v+\sigma^{p} \odot x .
$$

Replacing now $v$ by $-v$, it holds that $x$ is globalized robust against uncertainty in the profits, if there exist $v$ and $w$, such that $v, w, x$ satisfy

$$
\begin{aligned}
t-\sum_{i \in N} \overline{p_{i}} \sum_{j \in N^{+} \backslash\{i\}} x_{i j}+\rho_{A}\|v\|_{A}^{*}+\rho_{B}\|w\|_{B}^{*} & \leq 0, \\
w+v & =\sigma^{p} \odot x, \\
\|w\|_{C}^{*} & \leq \beta .
\end{aligned}
$$




\subsection{Formulation of the robust OP}

This subsection introduces the formulation of the ROP which incorporates both absolute robustness against fuel uncertainty and globalized robustness in the profit objective. The mathematical formulation reads

$$
(\mathrm{ROP}) \quad \max \left[\sum_{(i, j)=a \in A} x_{i j} \overline{p_{i}}-\rho_{A}\|v\|_{A}^{*}-\rho_{B}\|w\|_{B}^{*}\right],
$$

such that

$$
\begin{array}{rlrl}
\|w\|_{C}^{*} & \leq \beta, & & \\
w_{i}+v_{i} & =\sum_{j \in N^{+} \backslash\{i\}} \sigma_{i}^{p} x_{i j}=\left(\sigma^{p} \odot x\right)_{i}, & & \forall i \in N, \\
\sum_{(i, j)=a \in A} x_{i j} \overline{f_{i j}}+\sum_{s \in S} \rho_{s}\left\|y_{s}\right\|_{s}^{*} & \leq F, & & \\
\sum_{s \in S} y_{i j}^{s} & =\sigma_{i j}^{f} x_{i j}=\left(\sigma^{f} \otimes x\right)_{i j}, & & \\
\sum_{j \in N^{+} \backslash\{i\}} x_{i j}=\sum_{j \in N} x_{j 0} & =1, & & \\
\sum_{j \in N^{+} \backslash\{i\}} x_{j i} & \leq 1, & & \\
u_{i}-u_{j}+1 & \leq(1-j)=a \in A, \\
1 \leq u_{i} & \leq|N|, & & \\
x_{i j} & \in\{0,1\}, & & \forall i \in N, \\
y_{i j}^{s} & \in \mathbf{R}, & & \forall i, j \in N, \\
v_{i} & \in \mathbf{R}, & & \forall i \in N, \\
w_{i} & \in \mathbf{R}, & & \forall(i, j)=a \in A,
\end{array}
$$

\subsubsection{Simplifications}

Note that only the variables $x$ are integer. To ease the formulation of the norms in the Objective 35 and Constraints 36 and 38, the variables $v, w$ and $y_{s}$ can be restricted to the non-negative orthant. This is a consequence of the right hand side in Constraints 37 and 39 being non-negative. To prove this result, we will show that we can transform feasible vectors $y_{s} \in \mathbf{R}^{|A|}$ into feasible vectors $\bar{y}_{s} \in \mathbf{R}_{+}^{|A|}$. Let therefore vectors $y^{s} \in \mathbf{R}^{|A|}$ be given that satisfy the above constraints. Select $(i, j)=a \in A$ arbitrarily. Define the sets

$$
S^{-}=\left\{s \in S: y_{i j}^{s}<0\right\} \quad \text { and } S^{+}=\left\{s \in S: y_{i j}^{s} \geq 0\right\} .
$$

If $S^{-}=\emptyset$, the coordinates $y_{i j}^{s} \geq 0$ for all $s \in S$ and we can define $\bar{y}_{i j}^{s}=y_{i j}^{s}$ for all $s \in S$. Otherwise, as $\left(\sigma^{f} \otimes x\right)_{i j}$ is non-negative, it must hold that

$$
\sum_{s \in S^{+}} y_{i j}^{s} \geq-\sum_{s \in S^{-}} y_{i j}^{s}>0
$$

Define the quantity

$$
\gamma=\frac{\sum_{s \in S} y_{i j}^{s}}{\sum_{s \in S^{+}} y_{i j}^{s}}=\frac{\sum_{s \in S^{+}} y_{i j}^{s}+\sum_{s \in S^{-}} y_{i j}^{s}}{\sum_{s \in S^{+}} y_{i j}^{s}} .
$$

It follows from Equation 48, that $\gamma \in[0,1]$. Define now $\bar{y}_{i j}^{s}=0$ for all $s \in S^{-}$and $\bar{y}_{i j}^{s}=\gamma y_{i j}^{s}$ for all $s \in S^{\prime}$. We then have

$$
\sum_{s \in S} \bar{y}_{i j}^{s}=\sum_{s \in S^{+}} \bar{y}_{i j}^{s}=\gamma \sum_{s \in S^{+}} y_{i j}^{s}=\sum_{s \in S} y_{i j}^{s}=\left(\sigma^{f} \otimes x\right)_{i j} .
$$


Furthermore, it can easily be seen that $\left|\bar{y}_{i j}^{s}\right| \leq\left|y_{i j}^{s}\right|$. Repeating this procedure for all $(i, j)=a \in A$, we obtain vectors $\bar{y}^{s} \in \mathbf{R}_{+}^{|A|}$, that satisfy Constraint 39 for all $(i, j)=a \in A$ and that satisfy $\left|\bar{y}_{i j}^{s}\right| \leq\left|y_{i j}^{s}\right|$ for all $s \in S$ and $(i, j)$. For all $1 \leq p<\infty$, it now holds that

$$
\left\|\bar{y}^{s}\right\|_{p}^{p}=\sum_{(i, j)=a \in A}\left|\bar{y}_{i j}^{s}\right|^{p} \leq \sum_{(i, j)=a \in A}\left|y_{i j}^{s}\right|^{p}=\left\|y_{s}\right\|_{p}^{p} .
$$

We conclude that $\left\|\bar{y}^{s}\right\|_{p} \leq\left\|y_{s}\right\|_{p}$. Furthermore, it follows that

$$
\left\|\bar{y}^{s}\right\|_{\infty}=\max _{(i, j)=a \in A}\left|\bar{y}_{i j}^{s}\right| \leq \max _{(i, j)=a \in A}\left|y_{i j}^{s}\right|=\left\|y^{s}\right\|_{\infty} .
$$

As all norms that we consider are of one of the above forms, this implies that

$$
\left\|\bar{y}_{s}\right\|_{s} \leq\left\|y_{s}\right\|_{s} .
$$

We conclude that also Constraint 38 is satisfied. This proves that we can restrict the domains of $y_{s}$ to $\mathbf{R}_{+}^{|A|}$. A similar proof can be given to show that feasible vectors $v, w \in \mathbf{R}^{|N|}$ can be transformed into feasible vectors $\bar{v}, \bar{w} \in \mathbf{R}_{+}^{|N|}$ that give an objective value that is not worse. This proves that the auxiliary vectors $v, w$ and $y_{s}$ can be assumed to be non-negative.

\subsubsection{Selecting uncertainty sets}

The formulation just given, contains constraints based on the general uncertainty sets $Z^{f}, Z^{p}$ and $\Omega^{p}$. Here we will illustrate how to further specify these constraints for some specific choices of the uncertainty set. We will provide this illustration for two choices of the fuel uncertainty set: the $L^{1}$-ball and the intersection of the $L^{\infty}$-ball and the $L^{2}$-ball. Similar derivations hold for other fuel uncertainty sets, as well as for the sets representing the profit uncertainty. In these derivations we will need to specify dual norms. In order to do so, we will make use of the following known theorem on dual norms [2].

Theorem 1. If $p, q \in[0, \infty]$ satisfy $\frac{1}{p}+\frac{1}{q}=1$, then

$$
\|y\|_{p}^{*}=\sup \left\{y^{T} d:\|d\|_{p} \leq 1\right\}=\|y\|_{q} .
$$

\section{$L^{1}$-ball}

When we choose to define $Z^{f}$ as an $L^{1}$-ball, we have $Z^{f}=B_{1}^{|A|}\left(\rho_{1}\right)=\left\{\zeta \in \mathbf{R}^{|A|}:\|\zeta\|_{1} \leq \rho_{1}\right\}$. The fuel constraints given by Equations 38 and 39 then become

$$
\sum_{(i, j) \in A} \overline{f_{i j}} x_{i j}+\rho_{1}\left\|\sigma^{f} \otimes x\right\|_{1}^{*} \leq F
$$

since the index set $S=\{s\}$ in the definition of $Z^{f}$ is a singleton. From Theorem 1 we find $\|y\|_{1}^{*}=\|y\|_{\infty}$. Replacing $\left\|\sigma^{f} \otimes x\right\|_{1}^{*}$ by $\left\|\sigma^{f} \otimes x\right\|_{\infty}$ in Constraint 49 and introducing the auxiliary variable $y \in \mathbf{R}$ leads to the following reformulation

$$
\begin{array}{r}
\sum_{(i, j) \in A}\left\{\overline{f_{i j}} x_{i j}\right\}+\rho y \leq F, \\
y \geq \sigma_{i j}^{f} x_{i j} \forall(i, j) \in A, \\
y \geq-\sigma_{i j}^{f} x_{i j} \forall(i, j) \in A .
\end{array}
$$

Since both $\sigma_{i j}^{f}$ and $x_{i j}$ are nonnegative, the final constraint is redundant. This formulation implies that we apply a budget of $\rho$ times the maximum value over all $\sigma_{i j}^{f}$ 's selected in the tour, to serve as planned slack against deviations from the expected fuel usage. 


\section{Intersection $L^{\infty}$-ball and $L^{2}$-ball}

When we choose $Z^{f}$ to be the intersection of an $L^{\infty}$-ball and an $L^{2}$-ball, we have

$$
Z^{f}=B_{\infty}^{|A|}\left(\rho_{\infty}\right) \cap B_{2}^{|A|}\left(\rho_{2}\right)=\left\{\zeta \in \mathbf{R}^{|A|}:\|\zeta\|_{\infty} \leq \rho_{\infty},\|\zeta\|_{2} \leq \rho_{2}\right\} .
$$

The fuel constraints given by Equations 38 and 39 then become

$$
\begin{aligned}
\sum_{(i, j)=a \in A} x_{i j} \overline{f_{i j}}+\rho_{\infty}\left\|y_{\infty}\right\|_{\infty}^{*}+\rho_{2}\left\|y_{2}\right\|_{2}^{*} & \leq F \\
y_{i j}^{\infty}+y_{i j}^{2} & =\sigma_{i j}^{f} x_{i j}=\left(\sigma^{f} \otimes x\right)_{i j}, \quad \forall(i, j)=a \in A .
\end{aligned}
$$

From Theorem 1 we find $\|y\|_{\infty}^{*}=\|y\|_{1}$ and $\|y\|_{2}^{*}=\|y\|_{2}$. Replacing $\left\|y_{\infty}\right\|_{\infty}^{*}$ by $\left\|y_{\infty}\right\|_{1}$ and $\left\|y_{2}\right\|_{2}^{*}$ by $\left\|y_{2}\right\|_{2}$ in Constraint 53 and introducing the auxiliary variable $y \in \mathbf{R}$ and vector $z \in \mathbf{R}^{|A|}$ lead to the following reformulation

$$
\begin{array}{rlrl}
\sum_{(i, j)=a \in A} x_{i j} \overline{f_{i j}}+\sum_{(i, j)=a \in A} z_{i j}+y & \leq F, & \\
y_{i j}^{\infty}+y_{i j}^{2} & =\sigma_{i j}^{f} x_{i j}=\left(\sigma^{f} \otimes x\right)_{i j} & & \\
y & \geq \sqrt{\sum\left(y_{i j}^{2}\right)^{2}}, & & \\
z_{i j} & \geq y_{i j}^{\infty} & & \forall(i, j)=a \in A, j)=a \in A, \\
z_{i j} & \geq-y_{i j}^{\infty} & & \forall(i, j)=a \in A .
\end{array}
$$

The final constraint is again redundant, since $y_{i j}^{\infty} \geq 0$ for all $(i, j)=a \in A$, as we showed in Section 4.3.1. Therefore, these constraints can be reformulated in the following way:

$$
\begin{aligned}
\sum_{(i, j)=a \in A} x_{i j} \overline{f_{i j}}+\sum_{(i, j)=a \in A} y_{i j}^{\infty}+\sqrt{\sum\left(y_{i j}^{2}\right)^{2}} & \leq F, \\
y_{i j}^{\infty}+y_{i j}^{2} & =\sigma_{i j}^{f} x_{i j}=\left(\sigma^{f} \otimes x\right)_{i j} \quad \forall(i, j)=a \in A .
\end{aligned}
$$

\section{Case study}

In this section we will describe the computational experiments performed in order to solve the ROP. Real instances of AUV planning problems are, due to security reasons, restricted. Therefore we used representative data in order to illustrate the effects of applying robust optimization on a UAV planning problem. These data sets were used in earlier research on the OP ([7], [21], [11]), but since the standard OP is deterministic, the data sets do not include uncertainty parameters. Therefore, in the next subsection we describe how these data sets were adapted by introducing the uncertainty intervals for the fuel parameters and the profit parameters. Next, we will describe the shapes and sizes of the uncertainty sets for both the fuel parameters and the profit parameters. Finally, we will address the quality and reliability of the robust solution based on the combination of the uncertainty sets that were selected for the fuel and profit parameters. We also compare the probability that a solution is feasible for the robust solutions on one hand and the solution found with nominal fuel usage and nominal profits on the other.

\subsection{Nominal data}

The survey of the OP by Vansteenwegen et al. [22] contains an overview of benchmark instances for the OP. For our experiments we used the data set 'Tsiligrides problem 2' [21], which contains 20 nodes, each with a profit of either 15, 20, 25, 30, 40 or 50. The nodes are positioned on a rectangular area of size 15 by 15 units. We use the Euclidian distance $\operatorname{dist}(i, j)$ to represent the fuel requirement between node $i$ and $j$. Additionally, in order to realistically model the 
reconnaissance mission of the UAV, we add one parameter to this data set that represents the fuel required to properly record the target. That is, when the UAV has reached its target it may need to fly one or more orbits above the target in order to obtain all the information about the target requested for this mission. Equally for each of the nodes we set this fuel requirement to 2 units. Summarizing, $\overline{f_{i j}}$ is defined by $\operatorname{dist}(i, j)+2$ for all $(i, j) \in A \backslash\{(i, j) \mid j=s\}$. Note that the $\operatorname{arcs}(i, s)$, which are the arcs returning to the depot, only have a fuel requirement based on the Euclidean distance. We set the fuel capacity at 65 units.

\subsection{Uncertainty intervals}

Since the data set does not contain any uncertainty, we need to define the intervals within which we assume the realizations of both the fuel and the profit parameters to lie. That is, we need to define $\sigma_{i j}^{f}$ and $\sigma_{i}^{p}$ respectively.

\subsubsection{Fuel usage interval}

We assume the deviation in the fuel usage between the nodes to consist of two parts. Both are mainly due to deviations in the weather circumstances. The first part relates to the flight path by the UAV between the two points of interest. The second part models the fuel consumption required to capture full motion video or still imagery at the target location. For the first part, we assume the maximum absolute deviation of the fuel requirement to reach node $j$ from node $i$ to be a fixed percentage $\alpha^{f}$ of the nominal fuel usage $\operatorname{dist}(i, j)$. For the second part, capturing full motion video or still imagery, we assume a fixed maximum absolute deviation $c^{f}$ that is equal for all targets. Summarizing, we construct the interval of the fuel usage between node $i$ and node $j$ by defining $\sigma_{i j}^{f}=\alpha^{f} \operatorname{dist}(i, j)+c^{f}$. We choose $c^{f}=0.5$ and $\alpha^{f}=0.15$.

\subsubsection{Profit value interval}

We assume the uncertainty in the profit value to consist of two parts as well. First, we assume the realized profit value to depend on the expected profit value. More specifically, we assume the first part of the maximum absolute deviation between $p_{i}$ and $\overline{p_{i}}$ to be a fixed percentage $\alpha^{p}$ of the expected profit. Secondly, the size of the uncertainty interval might depend on the type of target. The mission planners might be able to specify the amount of uncertainty for a certain target location based on their knowledge about the target type and information obtained from previous missions. We model this variation in the level of uncertainty in the profit value between different targets, by introducing a random variable $U$. Summarizing, we define $\sigma_{i}^{p}=\alpha^{p} \overline{p_{i}}+U$, where we set $\alpha^{p}=0.2$ and we choose $U$ to be a random variable, uniformly distributed between 0 and 80 percent of the profit value.

\subsection{Uncertainty sets and parameters}

The robust counterpart of the $\mathrm{OP}$ requires three uncertainty sets to be defined: the sets defining all possible realizations of the fuel parameters $\left(Z^{f}\right)$, the set of realizations of the profits for which we want to maximize the worst case profit $\left(Z^{p}\right)$ and finally, the set for which we allow a slight loss in profit $\left(\Omega^{p} \supset Z^{p}\right)$. Additionally, for the globalized robust solution, a norm regarding the distance function, $\operatorname{dist}\left(\zeta, Z^{p}\right)$ needs to be defined. To define the uncertainty sets, we use balls that are defined with the $L_{1}$-norm, the $L_{2}$-norm and the $L_{\infty}$-norm. For the uncertainty in the fuel consumption, we also consider two intersections of balls: these intersections are defined with the $L_{\infty}$-norm and the $L_{1}$-norm and with the $L_{\infty}$-norm and the $L_{2}$-norm. For each of the three uncertainty sets, the associated size needs to be defined and for the distance function we require the constant $\beta$ to be defined. This leads to a total of eight settings that are required for any robust counterpart of the OP. 


\subsection{Computational experiments}

We implemented the robust counterpart of the ROP as formulated in Section 4.3. We used Eclipse 3.6 for the implementation and CPLEX 12.1 for the optimization of the OP on an Intel(R) Core(TM) 2 Duo CPU, $2.40 \mathrm{GHz}, 1.95 \mathrm{~GB}$ of RAM. As stated before, the robust counterpart requires eight settings to be defined. The tables below contain the settings of the experiments we performed. In our first set of experiments, we focus on the effect of implementing robustness in the fuel consumption. Here we ignore the uncertainty in the profits by setting the sizes of the associated uncertainty sets $\left(\rho_{A}\right.$ and $\left.\rho_{B}\right)$ to 0 . In the second set of experiments however, we concentrate only on the effect of globalized robustness in the profit objective and disregard the uncertainty in the fuel consumption by setting the size of the fuel uncertainty set to 0 .

In Table 1 we experiment with the use of different sizes and shapes of the fuel uncertainty set. Note that setting the size of $Z^{f}$ to $\rho=0$ corresponds to solving the nominal OP (since we also disregard profit uncertainty here), while $Z^{f}=B_{\infty}^{|A|}(1)$ corresponds to protection against all worst-case fuel realizations. Since protection against all worst-case realizations at the same time is a very conservative approach, we vary with several smaller uncertainty sets. The sets given in Table 1 are all inclusions of $Z^{f}=B_{\infty}^{|A|}(1)$. In Table 2 we gradually increase the size of the profit uncertainty sets $\Omega^{p} \supset Z^{p}$ and we experiment with the size of $\beta$. In all tables, the shape of the uncertainty set (denoted by index $p$ ) is not specified when its size is 0 .

To address the quality of the solutions produced by the different robust counterparts, we simulated realizations of both the fuel and the profit parameters. We executed 1000 runs in which all realizations were drawn randomly from a uniform distribution of the parameters within their interval and we evaluated the performance of each tour. In this way, we are able to determine the percentage of UAV tours that are feasible with respect to the fuel constraint. Also, we are able to determine an empirical distribution of the realized objective value (total priority value of visited targets) of the tour.

\begin{tabular}{|l|l|l|l|l|}
\hline$\Omega^{p}$ & $Z^{p}$ & $Z^{f}$ & dist & $\beta$ \\
\hline$B_{p}^{|N|}(0)$ & $B_{p}^{|N|}(0)$ & $B_{\infty}^{|A|}(\rho), \rho=[0,0.1, \ldots 0.9,1]$ & N.A. & 0 \\
$B_{p}^{|N|}(0)$ & $B_{p}^{|N|}(0)$ & $B_{1}^{|A|}(\rho), \rho=[0,0.1, \ldots 0.9,1]$ & N.A. & 0 \\
$B_{p}^{|N|}(0)$ & $B_{p}^{|N|}(0)$ & $B_{2}^{|A|}(\rho), \rho=[0,0.1, \ldots 0.9,1]$ & N.A. & 0 \\
$B_{p}^{|N|}(0)$ & $B_{p}^{|N|}(0)$ & $B_{\infty}^{|A|}(1) \cap B_{1}^{|A|}(\rho), \rho=[0,1, \ldots 9,10]$ & N.A. & 0 \\
$B_{p}^{|N|}(0)$ & $B_{p}^{|N|}(0)$ & $B_{\infty}^{|A|}(1) \cap B_{2}^{|A|}(\rho), \rho=[0,0.5, \ldots 2.5,3]$ & N.A. & 0 \\
\hline
\end{tabular}

Table 1: Varying size and shape of fuel uncertainty set.

\begin{tabular}{|l|l|l|l|l|}
\hline$\Omega^{p}$ & $Z^{p}$ & $Z^{f}$ & dist & $\beta$ \\
\hline$B_{\infty}^{|N|}\left(\rho_{A}\right), \rho_{A}=[0.1,0.2, \ldots 0.9,1]$ & $B_{\infty}^{|N|}\left(\rho_{B}\right), \rho_{B}=\rho_{A}-0.1$ & $B_{p}^{|A|}(0)$ & $L^{1}$ & 10 \\
$B_{2}^{|N|}\left(\rho_{A}\right), \rho_{A}=[0.1,0.2, \ldots 0.9,1]$ & $B_{1}^{|N|}\left(\rho_{B}\right), \rho_{B}=\rho_{A}$ & $B_{p}^{|A|}(0)$ & $L^{1}$ & 10 \\
$B_{\infty}^{|N|}(1)$ & $B_{\infty}^{|N|}\left(\rho_{B}\right), \rho_{B}=[0,0.1, \ldots 0.9,1]$ & $B_{p}^{|A|}(0)$ & $L^{1}$ & 10 \\
$B_{\infty}^{|N|}(1)$ & $B_{\infty}^{|N|}\left(\rho_{B}\right), \rho_{B}=0.2$ & $B_{p}^{|A|}(0)$ & $L^{1}$ & {$[0, \ldots 1000]^{*}$} \\
\hline
\end{tabular}

Table 2: Varying sizes of the profit uncertainty sets. ${ }^{*}$ For $\beta$ we used several elements ranging between 0 and 1000. The exact values can be found in Table 4 .

\subsection{Results}

The results of the computational experiments are summarized in Tables 3 and 4 which can be found in the Appendix. Table 3 contains the results of the experiments where we varied the size and shape of the fuel uncertainty set, as defined in Table 1. In Figure 1 the feasibility resulting from the simulation is plotted against the nominal objective value. This figure contains five lines. Each line corresponds to one of the shapes of the fuel uncertainty set and connects the results corresponding to different sizes. Table 4 contains the results of the experiments where we varied 
the sizes and shapes of the profit uncertainty sets as well as the size of $\beta$, as defined in Table 2 . For each combination of the profit uncertainty sets, we report several characteristics of the resulting empirical distribution. Figure 2 shows a histogram of the empirical distributions of the realized objective values for both the nominal tour and one of the globalized robust tours.

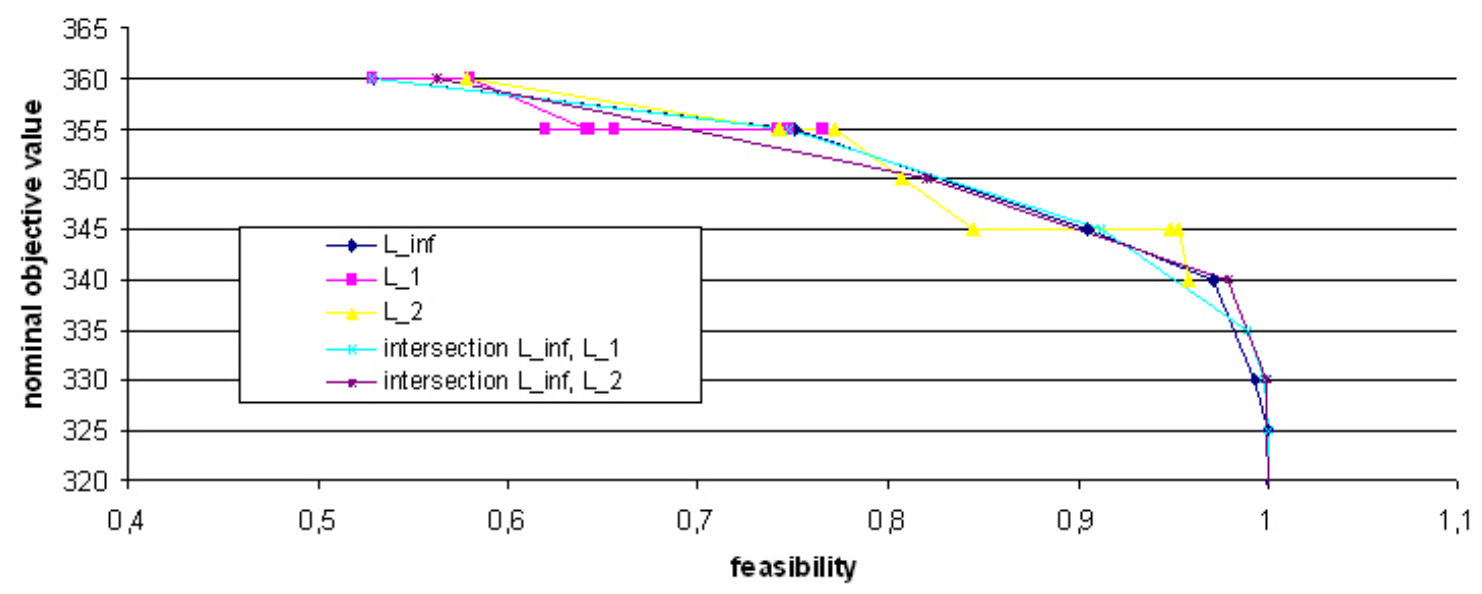

Figure 1: Fuel robustness: feasibility vs. nominal objective value

Table 3 illustrates how the profit value as well as the length of the tour decreases, when we gradually increase the size of the fuel uncertainty set. Increasing the size of the uncertainty set results in saving more planned slack of fuel. As such, a tour obtained by a large fuel uncertainty set has a lower expected profit on the one hand, but has a higher probability of remaining feasible on the other. This trade-off between feasibility and expected profit value is illustrated in Figure 1. Note that we cannot simply compare the nominal profit values of the different tours with one another. In reality, if during the flight the real fuel consumption turns out higher than expected, one or more targets on the tour might need to be canceled in order for the UAV to be able to return to its recovery point. Therefore, a robust tour is more likely to actually achieve the nominal target value, since the UAV is more likely to complete its predetermined flight plan.

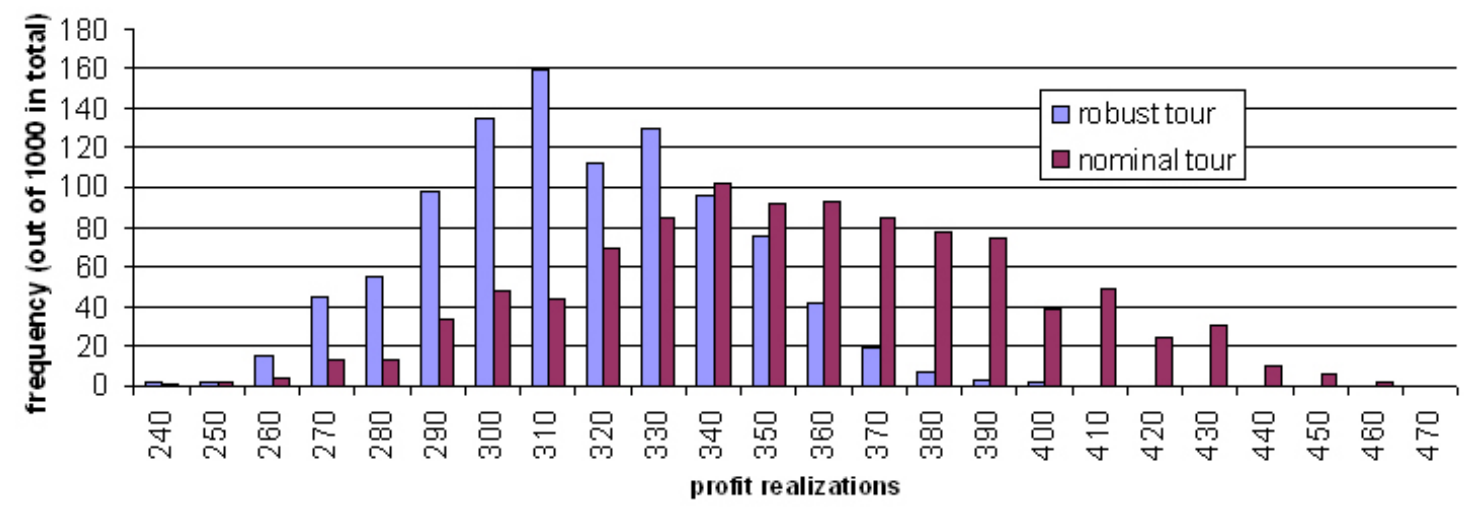

Figure 2: Distribution of profit realizations for the nominal tour and the globalized robust tour with $\Omega^{p}=B_{\infty}^{|N|}(1), Z^{p}=B_{\infty}^{|N|}(0.2)$ and $\beta=10$

With respect to the profit uncertainty, both Table 4 and Figure 2 show that globalized robustness provides solutions of which the variance of realizations is lower than the spread of realizations for the nominal tour. Globalized solutions having a relatively low standard deviation mainly occur 
when the set $\Omega^{p}$ is chosen relatively large and $\beta$ is not chosen too large. This is in line with our intuition: for smaller $\beta$, the solution is better protected against realizations in $\Omega^{p}$, which leads to solution with a lower standard deviation. The decrease in the expected (and realized) profit however, is relatively high compared to the decrease in standard deviation: with high probability the nominal tour gives a higher realized profit than a globalized robust tour. We expect this phenomenon to be the result of the discreteness of the solution space and the small size of the instance. Experimenting with larger instances becomes much more difficult, since solving the ROP requires not only a selection from the set of targets, but also the determination of a sequence in which the targets will be visited. On the other hand, the problem size used in this case study is representative for real-life instances of UAV mission planning. The computational results suggest that globalized robustness in the objective value does not produce encouraging results. They also suggest that a better way to deal with profit uncertainty in the objective function for small instances of the UAV planning problem might be using on-line planning approaches. On the other hand, robustness in the fuel constraint significantly improves the sustainability of the predetermined flight plan.

\section{Conclusion}

The optimization of information gathering in reconnaissance missions by UAVs can be modeled as an orienteering problem. Given the uncertainty in the military operational environment, robust planning solutions are needed. By applying techniques from robust optimization, we have introduced a new version of the orienteering problem that takes into account uncertainty in the parameters already in the modeling stage. We have shown how to find tours for the UAV that are robust against uncertainty in the fuel usage between targets as well as uncertainty in the importance of visiting specific target locations.

The results of the case study show that globalized robustness in the profit objective function is less applicable to our UAV planning problem. Regarding the fuel capacity on the other hand, incorporating robustness in the planning can significantly improve the sustainability of a predefined flight plan. In real-life UAV planning problems, when the fuel consumption turns out to be higher than accounted for, the UAV has to return to its recovery point earlier than planned. As a result, certain target information cannot be obtained anymore within that flight. Robust optimization allows the mission planner to overcome this issue by selecting a tour which balances the probability of infeasibility and the expected objective value, according to his or her preference.

\section{Acknowledgements}

In this paper we applied techniques from robust optimization, provided to us in an LNMB course by prof. A. Ben-Tal and prof. D. den Hertog. We greatly thank prof. A. Ben-Tal and prof. D. den Hertog for sharing their insights on the applicability of these techniques to our UAV planning problem.

\section{References}

[1] A. Ben-Tal and D. den Hertog. Robust optimization by Fenchel duality. Working paper, 2011.

[2] A. Ben-Tal, L. El Ghaoui, and A. Nemirovski. Robust Optimization. Princeton University Press, Princeton and Oxford, 2009.

[3] A. Ben-Tal and A. Nemirovski. Stable truss topology design via semidefinite programming. SIAM J. on Optimization, 7(4):991-1016, 1997.

[4] A. Ben-Tal and A. Nemirovski. Robust convex optimization. Mathematics of Operations Research, 23(4):769-805, 1998. 
[5] D. Bertsimas and M. Sim. The price of robustness. Operations Research, 52(1):35-53, 2004.

[6] L.F. Bertuccelli, M. Alighanbari, and J.P. How. Robust planning for coupled cooperative UAV missions. Decision and Control, 2004. CDC. 43rd IEEE Conference on, 14(4):5-19, 2004.

[7] I. Chao, B. Golden, and E. Wasil. Theory and methodology: a fast and effective heuristic for the orienteering problem. European Journal of Operational Research, 88:475-489, 1996.

[8] L. El Ghaoui and H. Lebret. Robust solution to least-squares problems with uncertain data. SIAM Journal on Matrix Analysis and Applications, 18:1035-1064, 1997.

[9] L. El Ghaoui, F. Oustry, and H. Lebret. Robust solutions to uncertain semidefinite programs. SIAM Journal on Optimization, 9:33-52, 1998.

[10] D. Feillet, P. Dejax, and M. Gendreau. Traveling salesman problems with profits: an overview. Transportation Science, 39:188-205, 2005.

[11] M. Fischetti, J. Salazar, and P. Toth. Solving orienteering problem through branch-and-cut. INFORMS Journal on Computing, 10:133-148, 1998.

[12] P. Kouvelis and G. Yu. Robust Discrete Optimization and its Applications. Kluwer Academic Publishers, London, 1997.

[13] G. Laporte and S. Martello. The selective traveling salesman problem. Discrete Applied Mathematics, 26:193-207, 1990.

[14] C. Miller, A. Tucker, and R. Zemlin. Integer programming formulations and travelling salesman problems. Journal of the ACM, 7:326-329, 1960.

[15] M. Poggi, H. Viana, and E. Uchoa. The team orienteering problem: Formulations and branchcut and price. 10thWorkshop on Algorithmic Approaches for Transportation Modelling, Optimization, and Systems (ATMOS10), pages 142-155, 2010.

[16] V. William Porto. Using evolutionary programming to optimize the allocation of surveillance assets. In Selected papers from the Second Asia-Pacific Conference on Simulated Evolution and Learning on Simulated Evolution and Learning, SEAL'98, pages 215-222, London, UK, 1999. Springer-Verlag.

[17] R.T. Rockafellar. Convex Analysis. Princeton University Press, Princeton, New Jersey, 1970.

[18] J. O. Royset and D. N. Reber. Optimized routing of unmanned aerial systems for the interdiction of improvised explosive devices. Military Operations Research, 3(4):2917-2922, 2010.

[19] Douglas A. Sammuelson. Changing the war with analytics. OR/MS Today, 37:30-35, 2010.

[20] A.L. Soyster. Convex programming with set-inclusive constraints and applications to inexact linear programming. Operations Research, 21(5):1154-1157, 1973.

[21] T. Tsiligrides. Heuristic methods applied to orienteering. Journal of the Operational Research Society, 35:797-809, 1984.

[22] P. Vansteenwegen, W. Souffria, and D. van Oudheusden. The orienteering problem: A survey. European Journal of Operational Research, article in press, 2010.

[23] Steve Zaloga. Unmanned Aerial Vehicles: Robotic Air Warfare 191\%-200\%. Osprey Publishing L, 2008.

\section{Appendix}




\begin{tabular}{|c|c|c|c|}
\hline fuel uncertainty set & nominal profit & nominal fuel consumption & feasibility \\
\hline$B_{\infty}^{|A|}(0)$ & 360 & 64.921 & 0.529 \\
\hline$B_{\infty}^{|A|}(0.1)$ & 355 & 63.662 & 0.751 \\
\hline$B_{\infty}^{|A|}(0.2)$ & 345 & 62.429 & 0.905 \\
\hline$B_{\infty}^{|A|}(0.3)$ & 340 & 61.403 & 0.971 \\
\hline$B_{\infty}^{|A|}(0.4)$ & 330 & 60.419 & 0.993 \\
\hline$B_{\infty}^{|A|}(0.5)$ & 325 & 59.315 & 1 \\
\hline$B_{\infty}^{|A|}(0.6)$ & 315 & 58.450 & 0.999 \\
\hline$B_{\infty}^{|A|}(0.7)$ & 310 & 57.361 & 1 \\
\hline$B_{\infty}^{|A|}(0.8)$ & 300 & 56.462 & 1 \\
\hline$B_{\infty}^{|A|}(0.9)$ & 290 & 55.734 & 1 \\
\hline$B_{\infty}^{|A|}(1)$ & 290 & 54.708 & 1 \\
\hline$B_{1}^{|A|}(0)$ & 360 & 64.921 & 0.529 \\
\hline$B_{1}^{|A|}(0.1)$ & 360 & 64.684 & 0.58 \\
\hline$B_{1}^{|A|}(0.2)$ & 360 & 64.684 & 0.58 \\
\hline$B_{1}^{|A|}(0.3)$ & 355 & 64.188 & 0.641 \\
\hline$B_{1}^{|A|}(0.4)$ & 355 & 64.417 & 0.619 \\
\hline$B_{1}^{|A|}(0.5)$ & 355 & 64.193 & 0.643 \\
\hline$B_{1}^{|A|}(0.6)$ & 355 & 64.213 & 0.656 \\
\hline$B_{1}^{|A|}(0.7)$ & 355 & 63.662 & 0.766 \\
\hline$B_{1}^{|A|}(0.8)$ & 355 & 63.610 & 0.742 \\
\hline$B_{1}^{|A|}(0.9)$ & 355 & 63.542 & 0.748 \\
\hline$B_{1}^{|A|}(1)$ & 355 & 63.542 & 0.748 \\
\hline$B_{2}^{|A|}(0)$ & 360 & 64.684 & 0.578 \\
\hline$B_{2}^{|A|}(0.1)$ & 355 & 63.610 & 0.743 \\
\hline$B_{2}^{|A|}(0.2)$ & 355 & 63.610 & 0.743 \\
\hline$B_{2}^{|A|}(0.3)$ & 355 & 63.610 & 0.742 \\
\hline$B_{2}^{\lceil A \mid}(0.4)$ & 355 & 63.594 & 0.772 \\
\hline$B_{2}^{|A|}(0.5)$ & 350 & 63.163 & 0.807 \\
\hline$B_{2}^{|A|}(0.6)$ & 345 & 62.963 & 0.845 \\
\hline$B_{2}^{|A|}(0.7)$ & 345 & 61.783 & 0.949 \\
\hline$B_{2}^{|A|}(0.8)$ & 345 & 61.783 & 0.953 \\
\hline$B_{2}^{|A|}(0.9)$ & 345 & 61.783 & 0.953 \\
\hline$B_{2}^{|A|}(1)$ & 340 & 61.641 & 0.959 \\
\hline$B_{\infty}^{|A|}(1) \cap B_{1}^{|A|}(0)$ & 360 & 64.921 & 0.529 \\
\hline$B_{\infty}^{|A|}(1) \cap B_{1}^{|A|}(1)$ & 355 & 63.542 & 0.748 \\
\hline$B_{\infty}^{|A|}(1) \cap B_{1}^{|A|}(2)$ & 345 & 62.315 & 0.912 \\
\hline$B_{\infty}^{|A|}(1) \cap B_{1}^{|A|}(3)$ & 335 & 60.794 & 0.989 \\
\hline$B_{\infty}^{|A|}(1) \cap B_{1}^{|A|}(4)$ & 330 & 59.829 & 0.998 \\
\hline$B_{\infty}^{|A|}(1) \cap B_{1}^{|A|}(5)$ & 325 & 59.035 & 1 \\
\hline$B_{\infty}^{|A|}(1) \cap B_{1}^{|A|}(6)$ & 310 & 57.591 & 1 \\
\hline$B_{\infty}^{|A|}(1) \cap B_{1}^{|A|}(7)$ & 310 & 57.361 & 1 \\
\hline$B_{\infty}^{|A|}(1) \cap B_{1}^{|A|}(8)$ & 305 & 56.624 & 1 \\
\hline$B_{\infty}^{|A|}(1) \cap B_{1}^{|A|}(9)$ & 295 & 55.816 & 1 \\
\hline$B_{\infty}^{|A|}(1) \cap B_{1}^{|A|}(10)$ & 290 & 54.945 & 1 \\
\hline$B_{\infty}^{|A|}(1) \cap B_{2}^{|A|}(0)$ & 360 & 64.684 & 0.562 \\
\hline$B_{\infty}^{|A|}(1) \cap B_{2}^{|A|}(0.5)$ & 350 & 63.110 & 0.82 \\
\hline$B_{\infty}^{|A|}(1) \cap B_{2}^{\lceil A \mid}(1)$ & 340 & 61.336 & 0.979 \\
\hline$B_{\infty}^{|A|}(1) \cap B_{2}^{\lceil A \mid}(1.5)$ & 330 & 59.762 & 0.999 \\
\hline$B_{\infty}^{|A|}(1) \cap B_{2}^{|A|}(2)$ & 315 & 58.383 & 1 \\
\hline$B_{\infty}^{|A|}(1) \cap B_{2}^{|A|}(2.5)$ & 305 & 56.624 & 1 \\
\hline$B_{\infty}^{|A|}(1) \cap B_{2}^{\lceil A \mid}(3)$ & 290 & 54.945 & 1 \\
\hline
\end{tabular}

Table 3: Results for varying sizes and shapes of $Z^{f}$ 


\begin{tabular}{|c|c|c|c|c|c|c|c|c|}
\hline$\Omega^{p}$ & $Z^{p}$ & $\beta$ & nominal profit & average & median & minimum & maximum & sd \\
\hline$B_{\infty}^{|N|}(0.1)$ & $B_{\infty}^{|N|}(0)$ & 10 & 360 & 361.24 & 360.03 & 250.57 & 483.57 & 40.74 \\
\hline$B_{\infty}^{|N|}(0.2)$ & $B_{\infty}^{|N|}(0.1)$ & 10 & 360 & 358.94 & 359.22 & 240.72 & 477.92 & 40.85 \\
\hline$B_{\infty}^{|N|}(0.3)$ & $B_{\infty}^{|N|}(0.2)$ & 10 & 360 & 359.68 & 358.62 & 247.96 & 496.07 & 41.48 \\
\hline$B_{\infty}^{|N|}(0.4)$ & $B_{\infty}^{|N|}(0.3)$ & 10 & 360 & 358.94 & 359.22 & 240.72 & 477.92 & 40.85 \\
\hline$B_{\infty}^{|N|}(0.5)$ & $B_{\infty}^{|N|}(0.4)$ & 10 & 360 & 359.68 & 358.62 & 247.96 & 496.07 & 41.48 \\
\hline$B_{\infty}^{|N|}(0.6)$ & $B_{\infty}^{|N|}(0.5)$ & 10 & 360 & 359.68 & 358.62 & 247.96 & 496.07 & 41.48 \\
\hline$B_{\infty}^{|N|}(0.7)$ & $B_{\infty}^{|N|}(0.6)$ & 10 & 345 & 345.02 & 344.45 & 245.70 & 480.14 & 38.48 \\
\hline$B_{\infty}^{|N|}(0.8)$ & $B_{\infty}^{|N|}(0.7)$ & 10 & 320 & 321.07 & 319.50 & 247.76 & 409.10 & 26.40 \\
\hline$B_{\infty}^{|N|}(0.9)$ & $B_{\infty}^{|N|}(0.8)$ & 10 & 320 & 320.43 & 319.31 & 242.77 & 409.32 & 26.44 \\
\hline$B_{\infty}^{|N|}(1)$ & $B_{\infty}^{|N|}(0.9)$ & 10 & 320 & 321.07 & 319.50 & 247.76 & 409.10 & 26.40 \\
\hline$B_{2}^{|N|}(0)$ & $B_{1}^{|N|}(0)$ & 10 & 360 & 359.68 & 358.62 & 247.96 & 496.07 & 41.48 \\
\hline$B_{2}^{|N|}(0.1)$ & $B_{1}^{|N|}(0.1)$ & 10 & 360 & 358.94 & 359.22 & 240.72 & 477.92 & 40.85 \\
\hline$B_{2}^{|N|}(0.2)$ & $B_{1}^{|N|}(0.2)$ & 10 & 360 & 359.68 & 358.62 & 247.96 & 496.07 & 41.48 \\
\hline$B_{2}^{|N|}(0.3)$ & $B_{1}^{|N|}(0.3)$ & 10 & 360 & 358.93 & 358.61 & 237.89 & 478.05 & 41.03 \\
\hline$B_{2}^{\lceil N \mid}(0.4)$ & $B_{1}^{|N|}(0.4)$ & 10 & 360 & 361.24 & 360.03 & 250.57 & 483.57 & 40.74 \\
\hline$B_{2}^{|N|}(0.5)$ & $B_{1}^{|N|}(0.5)$ & 10 & 360 & 358.93 & 358.61 & 237.89 & 478.05 & 41.03 \\
\hline$B_{2}^{|N|}(0.6)$ & $B_{1}^{|N|}(0.6)$ & 10 & 360 & 360.55 & 359.08 & 247.98 & 475.25 & 40.17 \\
\hline$B_{2}^{|N|}(0.7)$ & $B_{1}^{|N|}(0.7)$ & 10 & 360 & 359.68 & 358.62 & 247.96 & 496.07 & 41.48 \\
\hline$B_{2}^{|N|}(0.8)$ & $B_{1}^{|N|}(0.8)$ & 10 & 360 & 358.93 & 358.61 & 237.89 & 478.05 & 41.03 \\
\hline$B_{2}^{|N|}(0.9)$ & $B_{1}^{|N|}(0.9)$ & 10 & 360 & 358.93 & 358.61 & 237.89 & 478.05 & 41.03 \\
\hline$B_{2}^{|N|}(1)$ & $B_{1}^{|N|}(1)$ & 10 & 360 & 358.93 & 358.61 & 237.89 & 478.05 & 41.03 \\
\hline$B_{\infty}^{|N|}(1)$ & $B_{\infty}^{|N|}(0)$ & 10 & 320 & 320.68 & 321.06 & 244.56 & 403.96 & 26.62 \\
\hline$B_{\infty}^{|N|}(1)$ & $B_{\infty}^{|N|}(0.1)$ & 10 & 320 & 320.43 & 319.31 & 242.77 & 409.32 & 26.44 \\
\hline$B_{\infty}^{|N|}(1)$ & $B_{\infty}^{|N|}(0.2)$ & 10 & 320 & 321.07 & 319.50 & 247.76 & 409.10 & 26.40 \\
\hline$B_{\infty}^{|N|}(1)$ & $B_{\infty}^{|N|}(0.3)$ & 10 & 320 & 320.43 & 319.31 & 242.77 & 409.32 & 26.44 \\
\hline$B_{\infty}^{|N|}(1)$ & $B_{\infty}^{|N|}(0.4)$ & 10 & 320 & 321.07 & 319.50 & 247.76 & 409.10 & 26.40 \\
\hline$B_{\infty}^{|N|}(1)$ & $B_{\infty}^{|N|}(0.5)$ & 10 & 320 & 320.43 & 319.31 & 242.77 & 409.32 & 26.44 \\
\hline$B_{\infty}^{|N|}(1)$ & $B_{\infty}^{|N|}(0.6)$ & 10 & 320 & 321.07 & 319.50 & 247.76 & 409.10 & 26.40 \\
\hline$B_{\infty}^{|N|}(1)$ & $B_{\infty}^{|N|}(0.7)$ & 10 & 320 & 321.07 & 319.50 & 247.76 & 409.10 & 26.40 \\
\hline$B_{\infty}^{|N|}(1)$ & $B_{\infty}^{|N|}(0.8)$ & 10 & 320 & 320.43 & 319.31 & 242.77 & 409.32 & 26.44 \\
\hline$B_{\infty}^{|N|}(1)$ & $B_{\infty}^{|N|}(0.9)$ & 10 & 320 & 321.07 & 319.50 & 247.76 & 409.10 & 26.40 \\
\hline$B_{\infty}^{|N|}(1)$ & $B_{\infty}^{|N|}(1)$ & 10 & 320 & 321.07 & 319.50 & 247.76 & 409.10 & 26.40 \\
\hline$B_{\infty}^{|N|}(1)$ & $B_{\infty}^{|N|}(0.2)$ & 0 & 320 & 320.43 & 319.31 & 242.77 & 409.32 & 26.44 \\
\hline$B_{\infty}^{|N|}(1)$ & $B_{\infty}^{|N|}(0.2)$ & 0.01 & 320 & 321.07 & 319.50 & 247.76 & 409.10 & 26.40 \\
\hline$B_{\infty}^{|N|}(1)$ & $B_{\infty}^{|N|}(0.2)$ & 0.1 & 320 & 321.07 & 319.50 & 247.76 & 409.10 & 26.40 \\
\hline$B_{\infty}^{|N|}(1)$ & $B_{\infty}^{|N|}(0.2)$ & 1 & 320 & 321.07 & 319.50 & 247.76 & 409.10 & 26.40 \\
\hline$B_{\infty}^{|N|}(1)$ & $B_{\infty}^{|N|}(0.2)$ & 2 & 320 & 320.43 & 319.31 & 242.77 & 409.32 & 26.44 \\
\hline$B_{\infty}^{|N|}(1)$ & $B_{\infty}^{|N|}(0.2)$ & 5 & 320 & 320.43 & 319.31 & 242.77 & 409.32 & 26.44 \\
\hline$B_{\infty}^{|N|}(1)$ & $B_{\infty}^{|N|}(0.2)$ & 10 & 320 & 321.07 & 319.50 & 247.76 & 409.10 & 26.40 \\
\hline$B_{\infty}^{|N|}(1)$ & $B_{\infty}^{|N|}(0.2)$ & 20 & 335 & 334.90 & 334.42 & 242.99 & 434.82 & 30.92 \\
\hline$B_{\infty}^{|N|}(1)$ & $B_{\infty}^{|N|}(0.2)$ & 50 & 360 & 358.94 & 359.22 & 240.72 & 477.92 & 40.85 \\
\hline$B_{\infty}^{|N|}(1)$ & $B_{\infty}^{|N|}(0.2)$ & 100 & 360 & 358.94 & 359.22 & 240.72 & 477.92 & 40.85 \\
\hline$B_{\infty}^{|N|}(1)$ & $B_{\infty}^{|N|}(0.2)$ & 1000 & 360 & 359.68 & 358.62 & 247.96 & 496.07 & 41.48 \\
\hline
\end{tabular}

Table 4: Results Globalized Profit Robustness 